Proceedings

\title{
Cairsens $\mathrm{NO}_{2}$ : A Miniature Device Dedicated to the Indicative Measurement of Nitrogen Dioxide in Ambient Air ${ }^{\dagger}$
}

\author{
Brice Berthelot *, Azzédine Ben Daoud, Brice Hellio and Rony Akiki \\ Environnement S.A._Cairpol, 78304 Poissy CEDEX 4, France; brice.berthelot@esiee.fr (A.B.D.); \\ b.hellio@environnement-sa.com (B.H.); r.akiki@environnement-sa.com (R.A.) \\ * Correspondence: b.berthelot@environnement-sa.com; Tel.: +33-139-224-367 \\ + Presented at the Eurosensors 2017 Conference, Paris, France, 3-6 September 2017.
}

Published: 28 August 2017

\begin{abstract}
This paper reports the study of data quality of Cairsens $\mathrm{NO}_{2}$ environmental sensors. In order to evaluate sensors performances, the investigation was performed through: a prospective follow-up of short-term and long terms drifts, the evaluation of the effects of environmental conditions upon sensors measurements as well as the evaluation of the ozone-filter lifetime. In this purpose, a dedicated test bench has been developed to expose sensors to a fixed concentration of ozone while recording sensors response.
\end{abstract}

Keywords: low-cost gas sensor; indicative measurement; Air Quality Directive; ambient air pollution; nitrogen dioxyde

\section{Introduction}

Air pollution is the largest single environmental-health risk factor. In many European cities, air quality monitoring is currently performed thanks to a limited number of traditional fixed reference measuring stations and in compliance with EU legislation [1]. With the emergence of new paradigms which imply the use of small and cheaper devices, "Directive 2008/50/EC" suggests the use of indicative measurements. However these opportunities to improve air quality management and public health activities come with challenges mostly associated to data quality. Indeed, in real world applications, plenty of uncertain influences sources may generate discrepancies in sensors responses, which subsequently constitute as many sources of uncertainties. Mitigating effects of these sources of unreliability means in prior performing relevant and extensive tests to better identify use conditions, assets and limits of micro sensors technologies.

If such longstanding technical works are nowadays well-mastered for reference measurement methods, it is not the case for new low-cost sensors. Indeed, only few studies report relevant measurement data from low-cost sensor performance characterizations. That situation may be explained by the fact sources of uncertainties are various and lead to extensive characterization work. Such a situation leads to an obvious statement: the accurate characterization of increasingly-efficient devices require ever more in-depth studies which have a cost without any common measure with the price of low-cost instruments. In this context, this communication aims to assess the performances of Cairsens $\mathrm{NO}_{2}$. Here, we will focus on the laboratory performances characterization of devices, first step in order to qualify them as indicator.

\section{Materiel \& Methods}

Cairsens technology (Figure 1) consists in an integrated system that relies on an amperometric sensor, a dynamic air sampling, a patented filter and a low-noise electronic circuit which allows a 
direct display of the measured values and complete status with internal data logging. Reliability of the measurement is achieved by limiting the effect of humidity variations by using a gas specific inlet filter combined with dynamic air sampling system. For accurate $\mathrm{NO}_{2}$ monitoring, CairpolEnvironnement SA developed a filter allowing a complete removal of ozone in air while being weakly reactive to $\mathrm{NO}_{2}$.

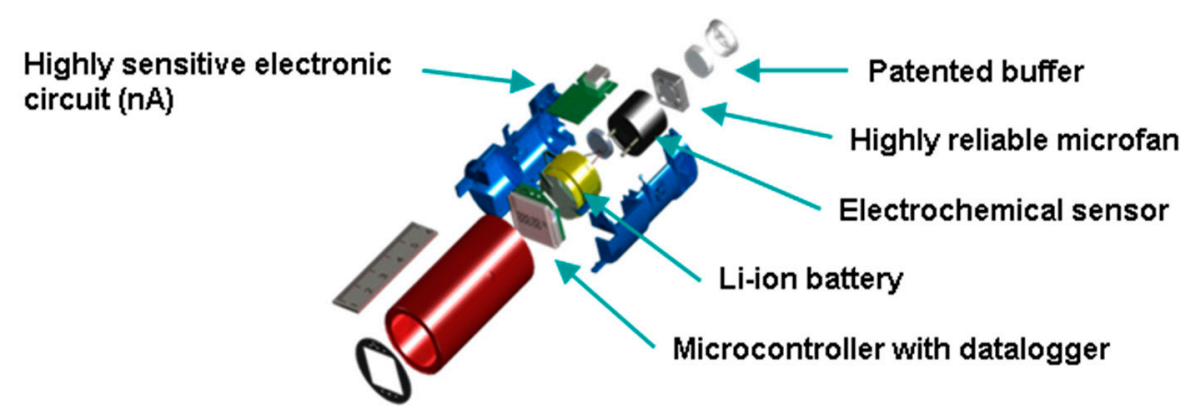

Figure 1. Exploded view of Cairsens $\mathrm{NO}_{2}$ technology.

A generic approach uncertainties estimation cannot be proposed due to the essentially different underlying mechanisms. Thus, our study has been divided into 3 parts: accuracy and drift evaluations; the evaluation of the effects of environmental conditions on sensors' responses; the evaluation of the selectivity of Cairsens $\mathrm{NO}_{2}$ towards its major interfering specie, ozone.

Regarding exposure chamber used for this characterization campaign, it is made of glass, as an inert material. Such a setup allows to easily change the concentration of the test gas and any interfering species while enabling a follow-up of temperature and relative humidity. Moreover, it should also be pointed out sensors have been prepared and mounted in a manner representative of the typical application, in accordance with our instructions manuals. Moreover, in order to avoid methodological bias and to get a representative picture of this technology, samples do not originate from the same production batch. By doing so, results presented in this communication take into consideration operator-dependent uncertainties during production phase and more precisely during calibration steps.

\subsection{Accuracy and Drift Evaluations}

As a first step of our work, Cairsens $\mathrm{NO}_{2}$ 's accuracy was qualified through classical metrological parameters:

- Linearity, in order to quantify the lack of fit of the sensor response;

- Limit of detection (LOD), determined from the standard deviation of repeatability at 0 ;

- Repeatability, computed from the standard deviation of repeatability at span;

- Instrumental drift, which is caused neither by a change in a quantity being measured nor by a change of any recognized influence quantity. That kind of discrepancy is associated to either the consumption/contamination of electrodes or changes of electrolyte properties.

Since it has been shown Cairsens $\mathrm{NO}_{2}$ does not suffer hysteresis effects, criteria cited above have been tested following the experimental design reported in Figure 2. Thus, $\mathrm{NO}_{2}$ has been tested at three different concentration levels (1: 0 ppb, 2: 125 ppb, 3: $225 \mathrm{ppb}$ ).

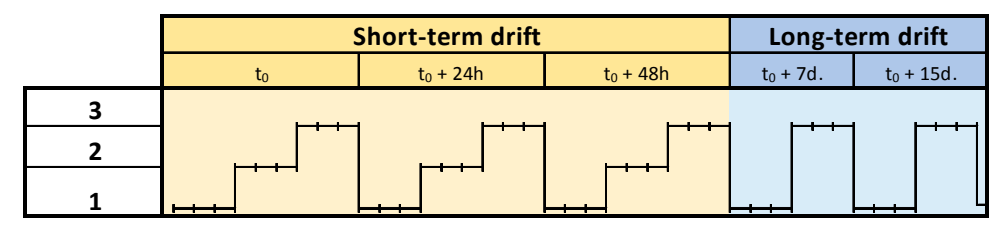

Figure 2. Experimental design considered for the evaluation of accuracy and drift of Cairsens $\mathrm{NO}_{2}$. 


\subsection{Effects of Environnemental Conditions}

Due to the fact electrochemical cells measurements lie on physicochemical and thermochemical processes, temperature and relative humidity are both key factors of uncertainties [2]. Thus, thanks to a climate chamber, decorrelated effects of temperature and relative humidity have been evaluated according the experimental design proposed in Figure 3.

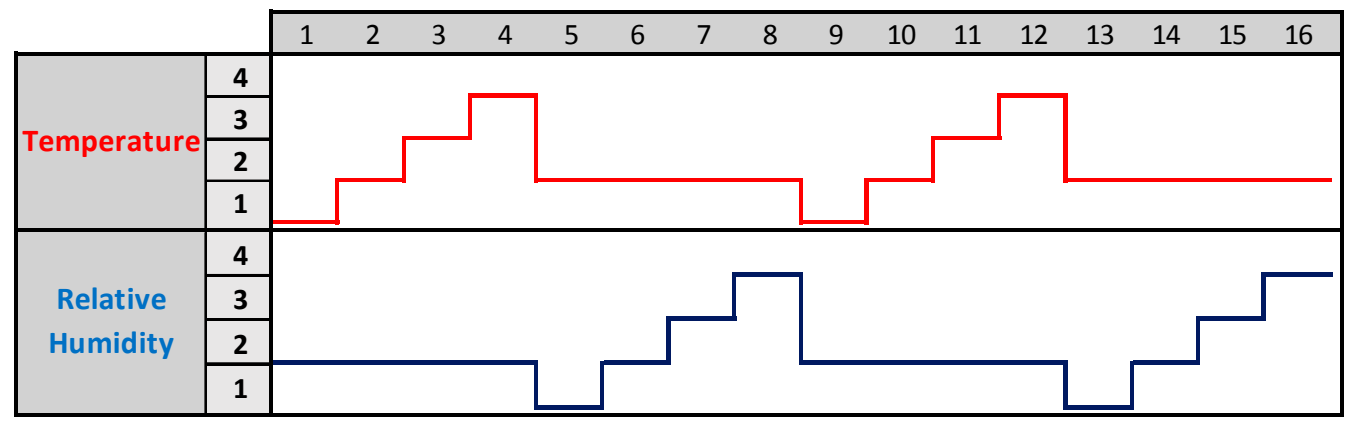

Figure 3. Sequence of trial tests for the evaluation of the effect of environmental conditions on the sensors responses. When temperature varies, relative humidity is maintained constant and vice-versa.

\subsection{Selectivity of Cairsens $\mathrm{NO}_{2}$}

Defined as the ability of the sensor to measure the analyte concentration while being insensitive towards potential interfering factor in atmosphere, the selectivity is a key parameter to evaluate the sensor performances. Regarding Cairsens $\mathrm{NO}_{2}$, the gas of interest for such considerations is ozone. Indeed, for accurate $\mathrm{NO}_{2}$ monitoring, Cairpol-Environnement $\mathrm{SA}$ developed a filter allowing a complete removal of ozone in air while being weakly reactive to $\mathrm{NO}_{2}$. In order to characterize the selectivity of our sensor, a gas-bench was designed to ensure a constant ozone concentration of 500 $\mathrm{ppb}$ in absence of $\mathrm{NO}_{2}$. A follow-up of the baseline response of the sensor is performed until the response reaches the $10 \mathrm{ppb}$. The time elapsed from the beginning of the experiment is so considered as the lifetime of the Cairsens $\mathrm{NO}_{2}$.

\section{Results}

\subsection{Accuracy and Drift Evaluations}

The results of ten Cairsens $\mathrm{NO}_{2}$ tested at various concentration levels are summarized on Table 1. As presented, the average value for the limit of detection is evaluated at $5.1 \pm 1.3 \mathrm{ppb}$, when the span drift after 15 days is below $0.5 \%$.

Table 1. Assessment of the accuracy and drift evaluation for 10 Cairsens $\mathrm{NO}_{2}$. Drifts are evaluated as the average of the absolute differences between consecutive pairs of measurements performed for the same concentration level and the same sensor.

\begin{tabular}{|c|c|c|c|c|c|c|c|c|c|c|}
\hline & S1 & S2 & S3 & S4 & S5 & S6 & S7 & S8 & S9 & $\mathrm{S} 10$ \\
\hline Linearity-Slope & 1.01 & 0.99 & 1.00 & 0.98 & 0.96 & 1.03 & 0.97 & 1.02 & 1.02 & 1.00 \\
\hline Linearity $-\mathrm{R}^{2}$ & 0.99 & 0.98 & 0.97 & 0.99 & 0.98 & 0.97 & 0.98 & 0.99 & 0.97 & 0.99 \\
\hline LOD (ppbv) & 6.0 & 6.7 & 6.0 & 4.8 & 5.3 & 6.8 & 4.0 & 4.0 & 3.0 & 4.5 \\
\hline Span repeteability (\%) & $3.9 \%$ & $8.4 \%$ & $6.2 \%$ & $4.1 \%$ & $5.8 \%$ & $7.8 \%$ & $4.3 \%$ & $4.4 \%$ & $3.5 \%$ & $5.1 \%$ \\
\hline Zero drift (ppbv) $-\mathrm{t}_{0}+48 \mathrm{~h}$ & 1.9 & 2.2 & 1.7 & 1.6 & 1.8 & 1.9 & 1.5 & 1.7 & 1.6 & 1.8 \\
\hline Zero drift (ppbv) $-t_{0}+15 d$ & 1.8 & 1.9 & 1.9 & 1.8 & 1.9 & 2.5 & 1.6 & 2.1 & 1.8 & 2.0 \\
\hline Span drift (ppbv) $-t_{0}+48 \mathrm{~h}$ & 2.4 & 2.7 & 2.7 & 2.2 & 3.3 & 2.7 & 3.7 & 4.9 & 2.3 & 3.5 \\
\hline Span drift $(\mathrm{ppbv})-\mathrm{t}_{0}+15 \mathrm{~d}$ & 3.3 & 3.6 & 3.2 & 3.1 & 5.3 & 4.2 & 3.9 & 6.1 & 3.1 & 4.7 \\
\hline
\end{tabular}




\subsection{Effects of Environnemental Conditions}

Each time, the results of five Cairsens $\mathrm{NO}_{2}$ tested at different environmental conditions are presented in Figure 4. These results have shown that the response-baseline drift due to temperature or relative humidity vary with an amplitude of $5 \mathrm{ppbv}$, which is lower than tolerated uncertainties for such kinds of sensors.

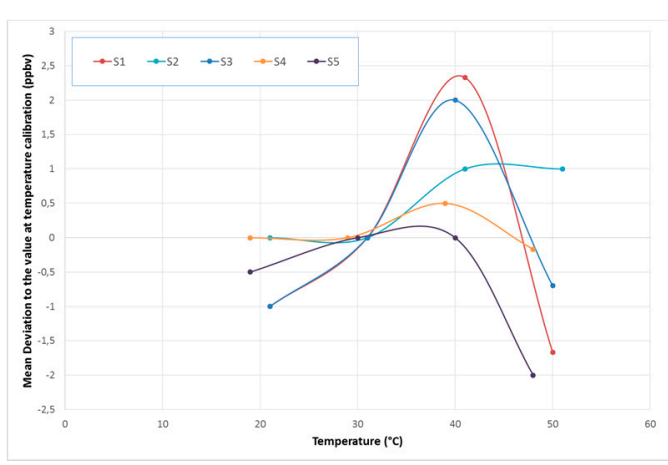

(a) Effects of temperature on sensors response

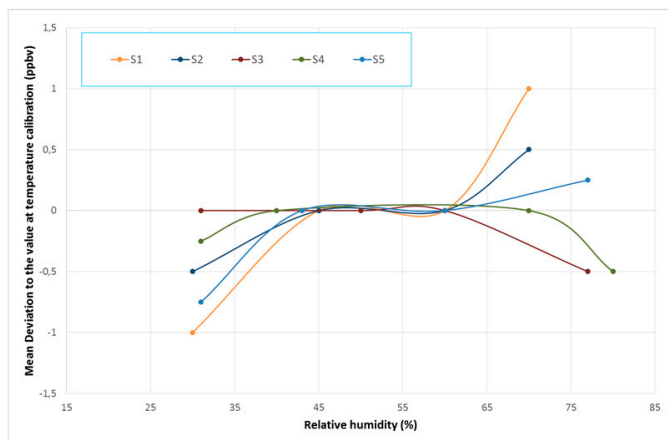

(b) Effects of relative humidity on sensors response

Figure 4. Response-baseline changes due to temperature and relative humidity.

\subsection{Selectivity of Cairsens $\mathrm{NO}_{2}$}

Ozone-filter lifetime has been evaluated using a dedicated gas bench in which ozone is generated at a concentration of $500 \mathrm{ppbv}$. The average time for Cairsens $\mathrm{NO}_{2}$ responses to reach 10 ppbv was evaluated at $17.3 \pm 1.5$ days. Considering Paris-like cities' background pollution, the lifetime of the ozone-filter is evaluated to more than 433 days [3].

\section{Conclusions}

Based on laboratory experiments, Cairsens $\mathrm{NO}_{2}$ have demonstrated interesting behaviors in terms of linearity of response, repeatability and drifts. The limit of detection was evaluated to below $10 \mathrm{ppbv}$ in laboratory conditions. This work of characterization have also shown that the influence of environmental conditions on Cairsens $\mathrm{NO}_{2}$ responses are limited. In addition, the ozone filter has demonstrated its efficiency by presenting a lifetime superior to one year for pollution conditions that can happen in Paris-like cities. Moreover, field trials have led to compare how accurate and reliable Cairsens $\mathrm{NO}_{2}$ are against reference methods. The results of this study will be detailed in the fulllength paper and will allow to discuss the compliance of Cairsens $\mathrm{NO}_{2}$ response with the Data Quality Objectives of indicative measurements set in the Air Quality Directive (2008/50/EC).

Conflicts of Interest: The authors declare no conflict of interest.

\section{References}

1. European Union. Directive 2008/50/EC of 21 May 2008 on Ambient Air Quality and Cleaner Air for Europe; European Union: Brussels, Belgium, 2008.

2. Helm, I.; Jalukse, L.; Leito, I. Measurement Uncertainty Estimation in Amperometric Sensors: A Tutorial Review. Sensors 2010, 10, 4430-4455, doi:10.3390/s100504430.

3. Airparif. Surveillance Et Information Sur La Qualité De L'air A Paris En 2014; AIR PARIF: Paris, France, 2015

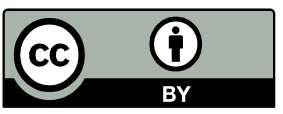

(C) 2017 by the authors. Licensee MDPI, Basel, Switzerland. This article is an open access article distributed under the terms and conditions of the Creative Commons Attribution (CC BY) license (http://creativecommons.org/licenses/by/4.0/). 